\title{
Research on Wireless Fading Characteristic in Urban Bridge Environment of the Inland Waterway Based on Channel Measurement
}

\author{
Jing Zhang ${ }^{1}$, Changzhen $\mathrm{Li}^{2}$, Xuanhao Shu ${ }^{2}$, Wei Chen ${ }^{2, *}$ \\ ${ }^{1}$ School of Automation, Wuhan University of Technology, Wuhan, China \\ ${ }^{2}$ School of Information Engineering, Wuhan University of Technology, Wuhan, China \\ Email address: \\ jzcaptain@whut.edu.cn (Jing Zhang),990244635@qq.com (Changzhen Li),626332858@qq.com (Xuanhao Shu), \\ greatchen@whut.edu.cn (Wei Chen) \\ *Corresponding author
}

\section{To cite this article:}

Jing Zhang, Changzhen Li, Xuanhao Shu, Wei Chen. Research on Wireless Fading Characteristic in Urban Bridge Environment of the Inland Waterway Based on Channel Measurement. International Journal of Wireless Communications and Mobile Computing. Vol. 7, No. 2, 2019, pp. 38-47. doi: 10.11648/j.wcmc.20190702.12

Received: January 28, 2020; Accepted: February 13, 2020; Published: February 20, 2020

\begin{abstract}
This paper focuses on the fading characteristics of a wireless channel of an inland waterway in an urban bridge scenario at $5.9 \mathrm{GHz}$. The measurement area was selected in Wuhan city, which lies on the middle reaches of the Yangtze River's intersection. Due to urban bridges, the fading characteristics of inland waterway channels are highly correlated with the ship motion direction or the distance between the transmitter and receiver and thus have unique properties. We demonstrated that the path loss, K-factor, power delay profile characteristics, and delay spread features significantly varied with the distance between the transmitter and receiver. Path-loss exponents were derived from the measurements and the differences between the Urban Bridge Environment and the line-of-sight was found. In bridge environments, the values of the excess delays change weakly from line-of-sight cases. The study also showed that numerical measurement results can be used to predict small-scale characteristics over any inland waterway with relatively good accuracy. These results will serve as a reference for urban waterways with bridges, as no experimental results have been reported previously.
\end{abstract}

Keywords: Urban Bridge Environment, Measurement, Channel Characteristics, Path Loss, Small Scale Fading

\section{Introduction}

With the rapid development of inland shipping, there has been increased focus on the characteristics of wireless channels in inland waterway environment [1]. Urban waterways have heavy ship traffic, various security requirements, numerous ships berthing at wharfs, and complex buildings on both sides of the waterway [2]. There have been many studies on the effects of different water surface environments, such as coastal water, inland river, and urban waterway environments, on wireless channels $[3,4]$. However, there have been few studies on the influence of urban bridges. This paper focuses on the impact of wireless propagation at $5.9 \mathrm{GHz}$ when ships travel across the urban bridges.

In the world, such as in China, there contains many bridges and various construction types [5]. The rapid development of bridges has greatly promoted city construction. Research on wireless propagation in urban bridge environment (UBE) may promote the construction of inland waterway communication. To alleviate the current lack of measurement data, we selected Wuhan city in China to perform UBE radio channel measurements and evaluate the path loss, power-delay profiles (PDP), and delay spread spectra for various types of ship motions in UBE.

To improve our understanding of the characteristics of UBE wireless fading, this study focused on channel fading for various types of ship motion in a UBE. There have been many studies on wireless propagation fading [6-8]. However, there is a significant difference between the wireless 
propagation in UBE and typical inland waterway scenario, especially in terms of channel characteristics. Several inland river wireless channel measurements have been performed to study the statistical properties of inland waterway propagation channels [9], nevertheless, channel characteristics that is suitable for UBE in inland waterway environments is still lacking. Previous inland waterway channel measurements have mainly focused on the fixed ship-to-land performance. For example, ITU-R Recommendation P. 1546-2 [10] gives a statistical and empirical method for predicting ship-to-land propagation losses; a multipath fading channel model was created based on data measured in the Aegean Sea. Previous experiments [11] demonstrated that distance has a significant impact on the PDP.

The aim of this study was to explore the relationship between ships and urban bridges. A classical methodology based on channel measurements was used [6]. This paper provides detailed descriptions of wireless communication for ships in UBE based on many measurements (Measurement ship travel across the urban bridge in different directions). The channel characteristics of ships with different types of motion near urban bridges were analyzed and compared based on the measured data.

This paper first gives a brief overview of the inland-waterway UBE. In Section II, we provide the details of the equipment and measurements. In Section III, the path-loss analysis used for the channel measurements is presented, and the data from three sets of measurements are given. The statistical feature analysis and crucial parameter estimations of small-scale fading are presented in Section IV. Finally, conclusions are provided in Section V.

\section{Environment, Equipment, and Measurements}

The measurements were obtained in the Yangtse river of the Wuhan section between the YingWu Zhou Bridge (YWZB) and the Wuhan Yangtze River Bridge (WHYRB). This type of bridge environment is common in inland river cities. Wireless channel propagation is easily disrupted by various factors, such as the presence of large building structures, when the ship is traveling under such conditions [12]. The measurement environment is shown in Figure 1. There are eleven bridges across the Yangtse River currently. This study focused on ships moving under the WHYRB. Figure 1 shows a satellite map of the UBE. The red dotted line corresponds to the YWZB, and the yellow dotted line corresponds to the WHYRB. The receiver (RX) station was located at the yellow position, which was 1500 and $550 \mathrm{~m}$ from the YWZB and WHYRB, respectively, with GPS coordinates of $30^{\circ} 30^{\prime} 51^{\prime \prime} \mathrm{N}$ and $114^{\circ} 17^{\prime} 15^{\prime \prime} \mathrm{E}$. In the measurement area, many anchored ships were observed along the riverside behind the RX. Thus, this area contained features that could significantly influence the channel fading characteristics during signal propagation.

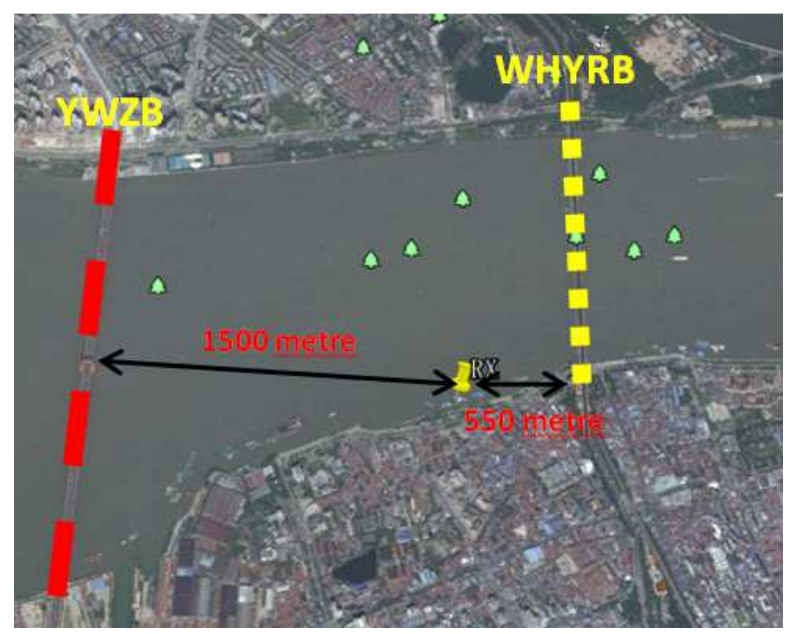

Figure 1. Satellite map of measured UBE area.

Measurements were obtained using a time-division multiplexing (TDM) channel sounder (Figure 2 (c) and (d)) provided by Super Radio AS of Norway to test the UBE channel characteristics. As shown in Figure 2, the channel sounder mainly consisted of the transmitter (TX) and RX (Figure 2 (a) and (b)). The parameters of the measurement setup are listed in Table 1. The TX antenna mounted on the ship (Figure 2 (b)) included an omnidirectional antenna pointed in the direction of the ship motion with a $2 \mathrm{~dB}$ gain. The RX was fixed on the deck of a pontoon mooring on the riverside (Figure 2 (a)). A chirp signal with a 10 ns delay resolution and a $100 \mathrm{MHz}$ bandwidth with a signal power of $16 \mathrm{~dB}$ was transmitted by the antenna.

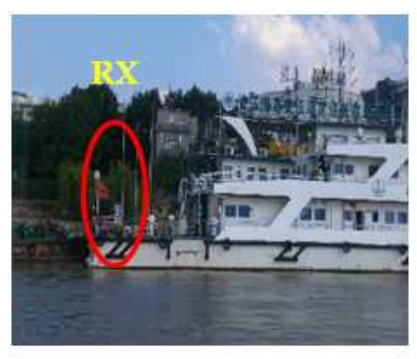

$a$

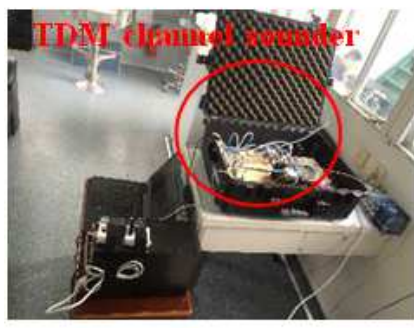

$c$

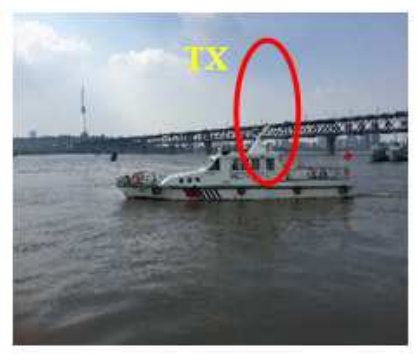

$b$

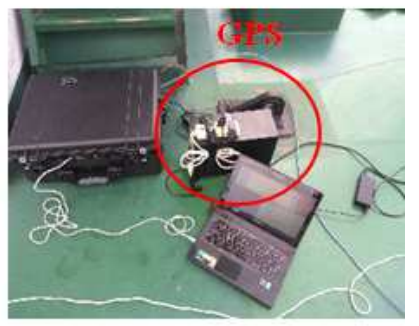

$d$
Figure 2. $a, b, c$, $d$ are photos of the propagation environments of $T X$ and $R X$ and channel sounder, respectively.

(a) RX position on the Pontoon.

(b) TX is mounted on the ship deck.

(c) TDM channel sounder.

(d) The remaining components of the test system. 
Moreover, the channel generation function $h(f, t)$ was obtained using a Fourier transform of the channel impulse response, and the maximum supporting Doppler band was $\pm 966.5 \mathrm{~Hz}$. Based on the time structure of the TDM channel sounder, the RX could receive 833 chirps per second $\left(T_{t}=\right.$ $1200 \mu \mathrm{s})$, and each chirp signal contained 2560 samples. The remaining components of the test system included laptops, power supplies, ships, a boat pontoon, and a global positioning system (GPS) (Figure 2 (c) and (d)).

Table 1. Measurement parameters.

\begin{tabular}{ll}
\hline Parameters & Values \\
\hline Center frequency & $5.9 \mathrm{GHz}$ \\
Delay resolution & $8.125 \mathrm{~ns}$ \\
Sampling/chirp & 2560 \\
TX Beam-width azimuth & Omni \\
TX Beam-width elevation & $\pm 5.5^{\circ}$ \\
TX antenna type & Dipole (vertical) \\
RX Beam-width elevation & $\pm 4^{\circ}$ \\
RX Beam-width azimuth & $\pm 45^{\circ}$ \\
TX height & $5.0625 \mathrm{~m}$ \\
RX height & $4.61 \mathrm{~m}$ \\
\hline
\end{tabular}

\subsection{Environment Description}

Bridge waterway: The UBE of an inland waterway contains a bridge that straddles the river, and ships pass under the bridge and move in the bridge environment. Due to the distance, the bridge, and other ships between the TX and RX, the line-of-sight (LOS) may be obstructed. To conduct the UBE study, three groups of method were selected, TX-B1, TX-B2, and TX-B3. To assess the effect of the bridges on the wireless propagation, we defined TX-B1 as the reference case for comparison with TX-B2 and TX-B3 and evaluate the channel fading in the UBE. To increase the reliability of the measurements, similar conditions (e.g., device parameters, distance between the TX and RX, and velocity) were tested for the TX-B1, TX-B2, and TX-B3. TX-B1 was on a ship moving along the river without any influence of the bridge, as shown in Figure 3. For TX-B2, the TX ship was moving downstream, and for TX-B3, the TX ship was moving upstream, as shown in Figure 3.

A schematic representation of Figure 3 with distances between the objects is shown in Figure 4. The RX was fixed between the two bridges during the measurements. The river had a width of 1100 meter. The distance between the RX and the trajectory of TX-B1 did not exceed 1750 meter, and the distances between the RX and the trajectories of TX-B2 and TX-B3 were less than 900 meter.

The velocity of RX was assumed to be zero. The TX with an omnidirectional antenna mounted on the ship moved with speed $\mathrm{v}$, and the water velocity in the Wuhan section in summer was assumed to be $2.5 \mathrm{~m} / \mathrm{s}$ [13]. Table 2 lists all the TX velocities.

Table 2. TX velocity data.

\begin{tabular}{ll}
\hline Status & TX Relative velocity $(\mathbf{m} / \mathbf{s})$ \\
\hline TX-B1 & [Min; Avg; Max] 6.8; $8 ; 9.2$ \\
TX-B2 & [Min.; Avg.; Max.] 6.8; 8.2; 9.7 \\
TX-B3 & [Min.; Avg.; Max.] 6.4; 7.2; 7.9 \\
\hline
\end{tabular}

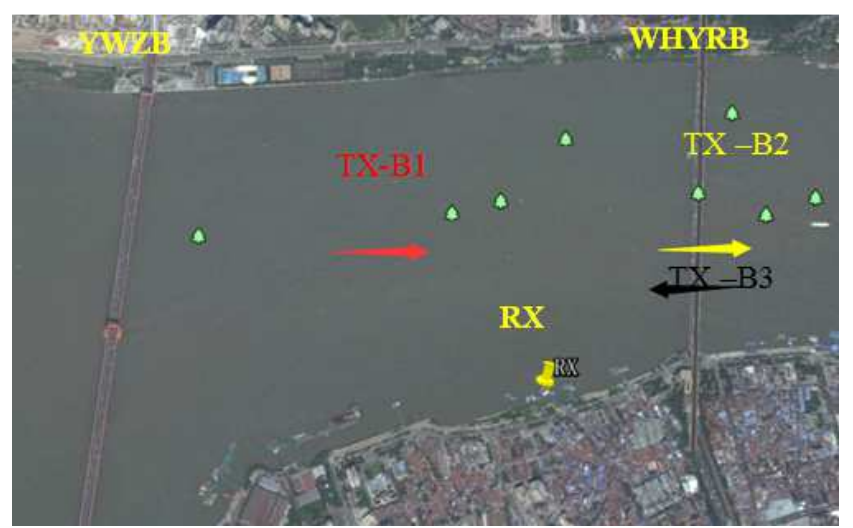

Figure 3. Google Earth map shows the investigated scenario in the IW.

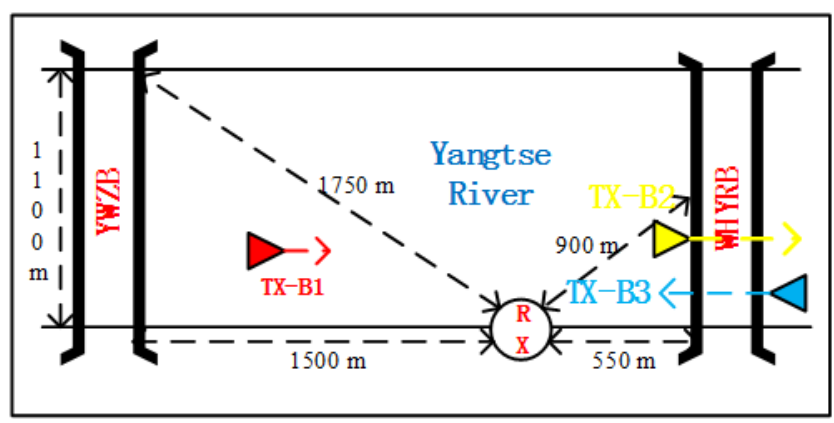

Figure 4. Overview of the measurement scheme.

\subsection{Data Collection and Processing}

To identify the channel statistical characteristics, the channel sounder signals were converted from the frequency domain to a channel impulse response (CIR) using a Fourier transform. The existing literature on CIRs is extensive and focuses particularly on the methods for collecting CIRs [14, 15]. For each measurement, a laptop can store the CIR values sampled by the RX, after which it collects the phase of each vector sample and the PDP of each associated received signal. Using the impulse response function of Bello [14], the relationship between the channel input and output can be expressed by an impulse response function.

$$
H(\tau)=\sum_{i=1}^{N} A_{i} e^{-j \varphi_{i} \delta\left(\tau-\tau_{i}\right)}
$$

where $H(\tau)$ is known as the time-varying impulse response function. $\mathrm{A}_{\mathrm{i}}$ is the attenuation amplitude of path $\mathrm{i}, \varphi_{i}$ is the phase deviation of path $\mathrm{I}, \tau_{i}$ is the time delay of path $\mathrm{i}, \mathrm{N}$ is the total number of paths, and $\delta_{\tau}$ is the Dirac Delta function. The channel is typically characterized using the autocorrelation function of the impulse response

\section{Path Loss}

The derivation of the path loss is determined using the measured transfer functions. From previous environment descriptions, we first compiled each measurement location channel's statistical descriptions. To determine the path loss, 
the time-varying PDP was derived for each time sample. For each CIR matrix, the PDP was described for each row as follows.

$$
P(n, \mu)=\sum_{t=0}^{t=i}|g(n, m)|^{2} \delta(\mu-m)
$$

where $\mathrm{n}$ is the index of the row, $\mu$ is the index of the discrete delay, and $g(n, m)$ is the CIR matrix that represents the element in the $\mathrm{n}^{\text {th }}$ row and $\mathrm{m}^{\text {th }}$ column. Each PDP was normalized by the strongest tap, and average the PDP was computed by averaging all the normalized PDPs in the CIR matrix. The averaged PDP (APDP) was calculated as follows [16]:

$$
P_{h}\left(t_{j}, \tau\right)=N A V^{-\sum_{n=0}^{N A V-1}\left|h\left(t_{j}+n \Delta t, \tau\right)\right|^{2}}
$$

In this expression, $h\left(t_{j}+n \Delta t, \tau\right)$ is defined by Equation (1), and:

$$
t_{j}=\left(0, N A V \Delta t, \ldots,\left\lfloor\frac{N_{t}}{N A V-1}\right\rfloor N A V \Delta t\right)
$$

where $\tau$ is the propagation delay, NAV is the time sample value for small scale fading, and $\Delta t$ is the time step. NAV was calculated as $\mathrm{s} / \mathrm{v} \Delta \mathrm{t}$, where $\mathrm{v}$ is the velocity of the TX, as shown in Table 2, and $\mathrm{s}$ is the wavelength that corresponds to the movement.

Finally, the antenna influence and some of the cable attenuation were removed from the measurement gain data. As the propagation distance increased, the overall path loss increased. These loss factors may affect the communication channel [17], and the path loss formula is expressed as follows:

$$
P L(D)=10 d B+G_{t}+G_{r}-P_{r}
$$

where PL (d) is the path loss at distance $d, d$ is the distance between the RX and TX, $10 \mathrm{~dB}$ is the sum of the transmission power of the wireless signals and the cable transmission loss, $G_{t}$ is the transmitted antenna gain, $G_{r}$ is the received antenna gain, and $\mathrm{P}_{\mathrm{r}}$ is the received signal power at distance $d$. The path loss value and the average path loss of each test point can be measured.

Figure 5 illustrates some of the main characteristics of the path loss when the ship was in an UBE. Figure 5 (a) shows the path loss when there was no bridge between the RX and TX (TX-B1 case), and the path loss increased with distance. Obstacles and distance increased the path loss. The signal power for TX-B1 ranged from 87.34 to $92.29 \mathrm{~dB}$, and the mean value was $89.96 \mathrm{~dB}$. There was a significant positive correlation between path loss and distance for the LOS case.

The path loss of TX-B2 did not increase with distance, as shown in Figure 5 (b). The differences between Figure 5 (a) and 5 (b) are significant. The path loss value decreased significantly from 92.28 to $91.03 \mathrm{~dB}$ when the ship was under the bridge. After the ship moved passed the bridge, the path loss value steadily increased from 91.03 to $92 \mathrm{~dB}$ as the spacing between the TX and RX increased. The findings for TX-B1 and TX-B2 indicate that large obstacles can affect the propagation features in inland waterways. The path loss rate probably fluctuated dramatically at the same distance, and the path loss appeared to be affected by bridge.

Figure 5 (b) and (c) showed significant differences. The path loss of TX-B2 was about 7.27 dB higher than of TX-B3 on average, as shown in Table 3. The lowest path loss value appeared under the bridge, as shown in Table 4. The differences between TX-B2 and TX-B3 are highlighted in Table 4, and the path loss on TX-B2 was about $7.99 \mathrm{~dB}$ higher than on TX-B3 on average according Table 3. These results confirmed that the bridge affected wireless propagation, as the path loss sharply decreased at the bridge position. The greater path loss of TX-B2 compared to TX-B3 could be attributed to various factors, such as the antenna mounting locations on the ship or channel diversity and shadow fading due to the direction of motion and position of the ship.
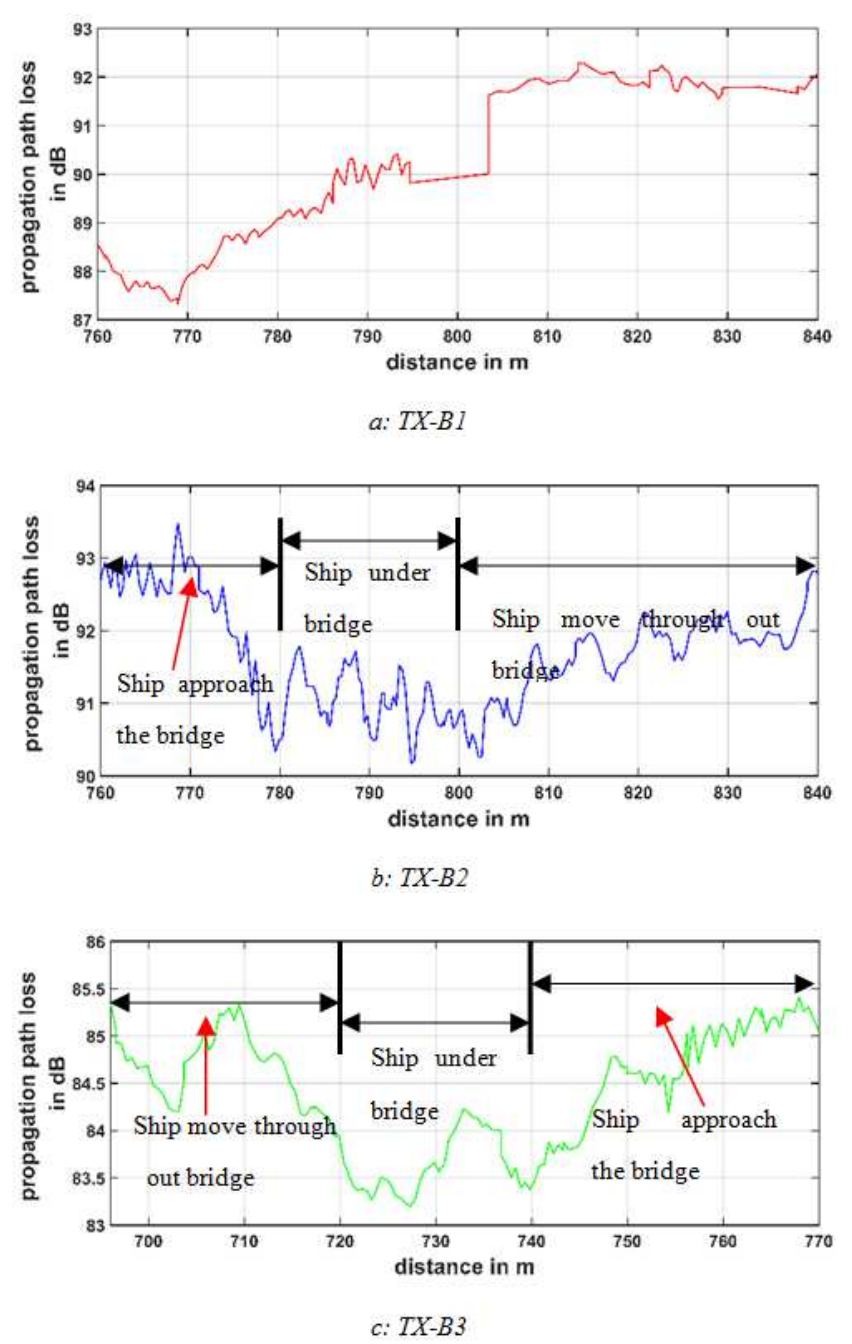

Figure 5. Propagation path loss analysis.

(a) TX-B1: No affected by bridge for reference, ship move along the river.

(b) TX-B2: Affected by bridge, ship moving downstream.

(c) TX-B3: Affected by bridge, ship moving upstream. 
Table 3. The Statistical Characteristics of Path Loss in whole measurement.

\begin{tabular}{lllll}
\hline States & Min & Max & Ave & Std \\
\hline TX-B1 & 87.34 & 92.29 & 89.96 & 1.66 \\
TX-B2 & 90.17 & 93.48 & 91.64 & 0.73 \\
TX-B3 & 83.20 & 85.40 & 84.37 & 0.62 \\
\hline
\end{tabular}

Table 4. The Statistical Characteristics of Path Loss when ship under bridge.

\begin{tabular}{lllll}
\hline States & Min & Max & Ave & Std \\
\hline TX-B2 & 90.17 & 91.79 & 91.63 & 0.37 \\
TX-B3 & 83.20 & 84.23 & 83.64 & 0.30 \\
\hline
\end{tabular}

\section{Small Scale Fading Characteristics}

Small-scale fading is a rapid change in the amplitude, phase, or multipath delay when the radio signal is transmitted over a short time or distance [18]. Many researchers have utilized small-scale fading to measure the channel characteristics. To obtain the small-scale characteristics, the path losses were filtered from the received signals. In this article, the small-scale fading used a sliding window with a length of 20 wavelengths to study and analyze the raw data.

\subsection{Amplitude Fading Distribution}

The Akaike information criterion (AIC) standard was founded and developed by Japanese statistician Akio Hiroshi [19]. It is a method used to measure the goodness of a statistical model fit. The AIC information can estimate the complexity of a model and use it to compare common channel distributions, such as the Rician, Rayleigh, and Nakagami distributions [20-22]. Moreover, the AIC information can be used to obtain fitting curves of the measurement data to rule out the possibility of overfitting. Therefore, based on the smallest AIC value method, the optimum preferred model can be selected. Normally, the AIC is calculated as follows:

$$
A I C=e^{\left(\frac{2 k}{T}\right)} \frac{\sum_{t=1}^{T} e_{t}^{2}}{T}
$$

where $e^{2 k / T}$ is the penalty factor, and $\mathrm{k}$ is the number of coefficients, $\mathrm{T}$ is number of the sample observed. It was further assumed that the error of the model obeyed an independent normal distribution, and thus the AIC can be expressed as follows:

$$
\ln A I C=\frac{2 k}{T}+\ln \left(\frac{R S S}{T}\right)
$$

where RSS is the root sum squared. If RSS is applied, it is usually represented by a normal or approximately normal distribution. The RSS is equal to the square root of the sum of the squares of the measurement statistics.

The results confirmed the association between the AIC values and the effects of various distributions on the channel propagation. Figure 6 below illustrates some of the main characteristics of the amplitude distribution values for these test periods. The red " $\times$ " markers denote the Rician distribution, the blue " + " markers denote the Nakagami-m distribution, the green " $\star$ " markers denote the Rayleigh distribution, and the black " $\nabla$ " markers denote the Weber distribution. The results of the AIC distribution correlational analysis are shown in Figure 6 (a), (b) and (c) and Table 5.

As shown in Table 5 and Figure 6, the distributions of TX-B1 and TX-B3 were more Rician than the TX-B2 group. The Rician distribution was the most prominent due to the absence of obstructions between the TX ship and RX in the TX-B1 and TX-B3 groups. Thus, there was always a LOS between the TX and the RX. In TX-B2 case, the Rayleigh distribution was mainly present in the absence of any direct path when the radio waves could not be directly transmitted to the RX. When the TX and RX were within the non-line-of-sight (NLOS) environment, the Rayleigh distribution was more prominent. The most surprising aspect of the data is the inconsistency between TX-B2 and TX-B3. There are several possible explanations for this result. One explanation is that there was no relationship between the channel distribution and bridge. This rather contradictory result may have been caused by other factors. Thus, some factors, such as the ship's motion direction or the mounting position of the TX on the ship, must be examined.

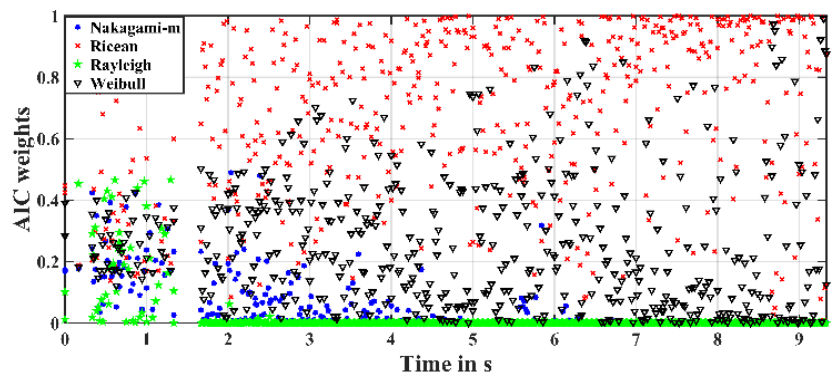

a: AIC distribution of TX-B1.

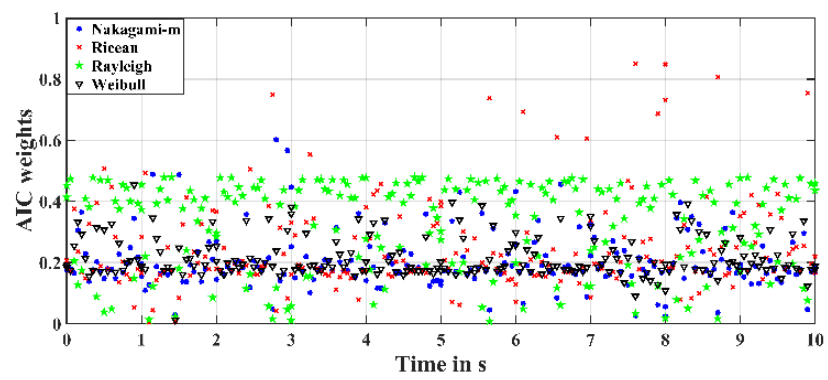

b: AIC distribution of TX-B2.

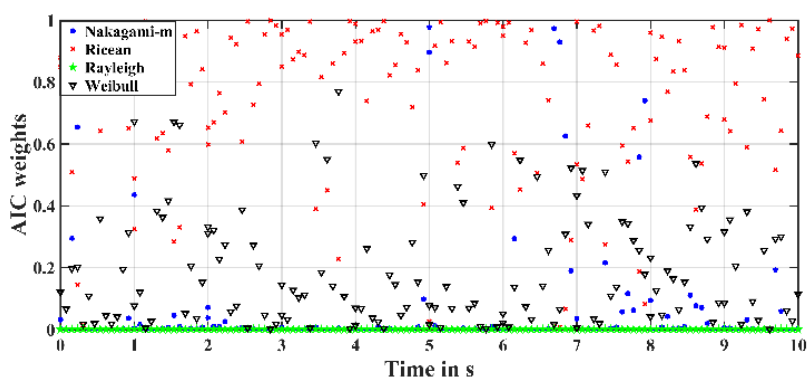

c: AIC distribution of TX-B3.

Figure 6. AIC distribution. 
Table 5. The Proportion of Distributions in Different States.

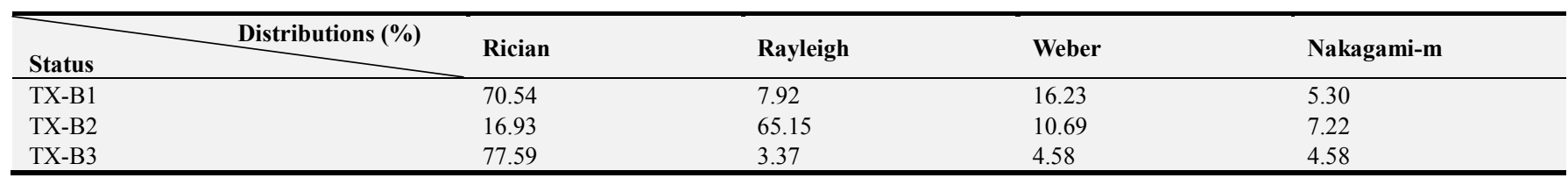

\subsection{The Rician Distribution K-Factors Analysis}

The Rician distribution is a continuous probability distribution that was used to quantify the time-varying characteristics of the received signal envelope in the wireless channel. The probability density function of the Rician distribution is expressed as follows:

$$
P(r)=\frac{r}{\sigma^{2}} e^{\left(\frac{r^{2}+A^{2}}{-2 \sigma^{2}}\right) \cdot I_{0}\left(\frac{r A}{\sigma^{2}}\right)}
$$

where $r$ is the envelope of the received signal, $\sigma^{2}$ is the variance of the multipath components, $A$ is the power of the main signal, and $I_{0}$ is the zeroth-order modified Bessel function of the first kind.

The K-factor is one of the most common parameters for evaluating a Rician distribution. It can be defined as the ratio of the main signal power and the variance of the multipath components as follows:

$$
K=\frac{A^{2}}{2 \sigma^{2}}
$$

To better understand the K-factor, we classified the expression into the $\mathrm{dB}$ type as follows:

$$
K=10 \log \frac{A^{2}}{2 \sigma^{2}}
$$

When $\mathrm{A}=0, \mathrm{~K}=-\infty \mathrm{dB}$, which indicates that the multipath fading is the largest, and the Rician distribution will transform to a Rayleigh distribution. When $\mathrm{K}=\infty \mathrm{dB}$, signal fading is not present. Moreover, if the multipath component value is very high, the $\mathrm{K}$-factor value will be reduced. When LOS propagation is present in the multipath propagation, the multipath signal obeys a Rician distribution. If the transmission path between the TX and $\mathrm{RX}$ is blocked by an object, the propagation path will convert from LOS to NLOS. For the Rician distribution, the K-factor defines the ratio of the power of the main signal to the variance of the multipath component. In the LOS channel, the K-factor is generally positive, whereas if there is a reflection path or NLOS channel, the K-factor becomes negative.

\subsubsection{The K Factor Estimation in TX-B1}

To obtain the $\mathrm{K}$ factor in TX-B1, we examined the TX-B1 data, which was used as a reference for assessing the quality of the other channels. In this experiment, the LOS was observable and could be detected along the entire inland waterway (Figure 7). The maximum $\mathrm{K}$-factor was $7.52 \mathrm{~dB}$, the minimum value was $-2.19 \mathrm{~dB}$, the mean was $4.57 \mathrm{~dB}$, and the Std was 2.04.
Thus, in the TX-B1 state, the channel experienced slight fading, and the LOS path was strong. LOS propagation was completely dominant throughout the entire measurement.

To distinguish between these two groups with similar propagation processes, the $\mathrm{K}$ factors were calculated and are shown in Figure 8. The performances of TX-B2 and TX-B3 were compared with TX-B1, and significant differences were identified. The $\mathrm{K}$ factors of the TX-B1 and TX-B2 cases were estimated using the same displacement distance and motion direction. There was no obstacle interference to the LOS for the TX-B1. However, the $\mathrm{K}$ factor of TX-B2 was extremely small, which suggested there was considerable multipath fading during propagation.

\subsubsection{The $K$ Factor Estimation in TX-B2 and TX-B3 State}

The TX-B2 and TX-B3 performances were compared. Although the $\mathrm{K}$ factor of TX-B2 was extremely weak, TX-B2 exhibited the largest $\mathrm{K}$ factor. This result is somewhat counterintuitive. This inconsistency may have been due to various factors, including the ship motion direction and the mounting position of the TX on the ship. This also explains that the bridge was not a major factor during wireless propagation.

Table 6. The Statistical Characteristics of K factor in two measurement.

\begin{tabular}{lllll}
\hline States & Min & Max & Ave & Std \\
\hline TX-B2 & $-2.24 \mathrm{~dB}$ & $0.39 \mathrm{~dB}$ & $-1.14 \mathrm{~dB}$ & 0.73 \\
TX-B3 & $1.53 \mathrm{~dB}$ & $6.51 \mathrm{~dB}$ & $4.73 \mathrm{~dB}$ & 0.95 \\
\hline
\end{tabular}

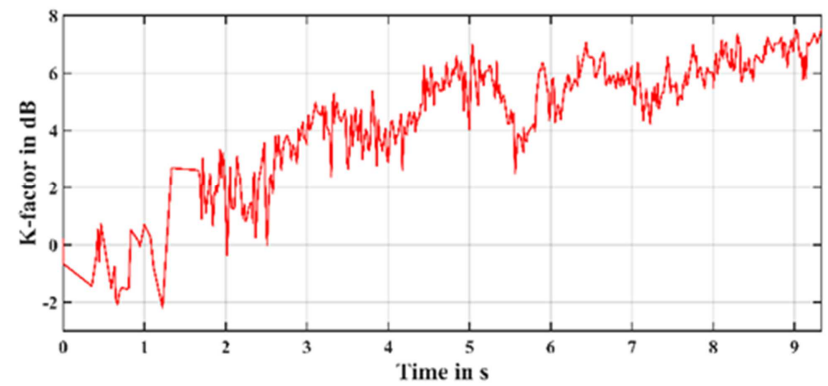

Figure 7. Test scenario Rician distribution $K$-factor in $T X-B 1$.

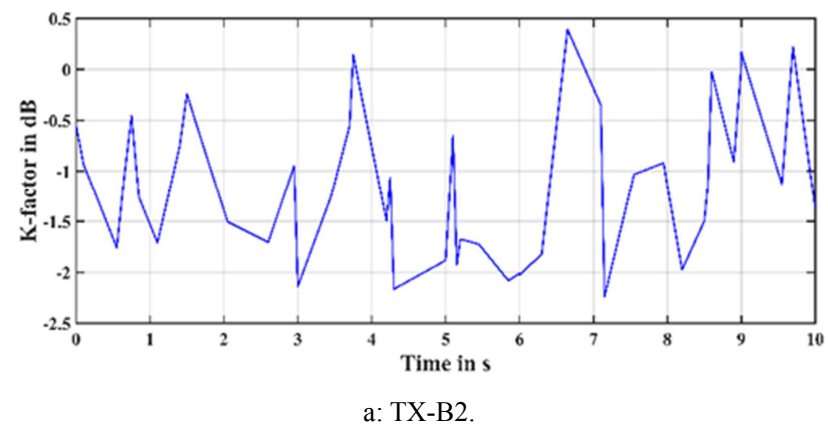




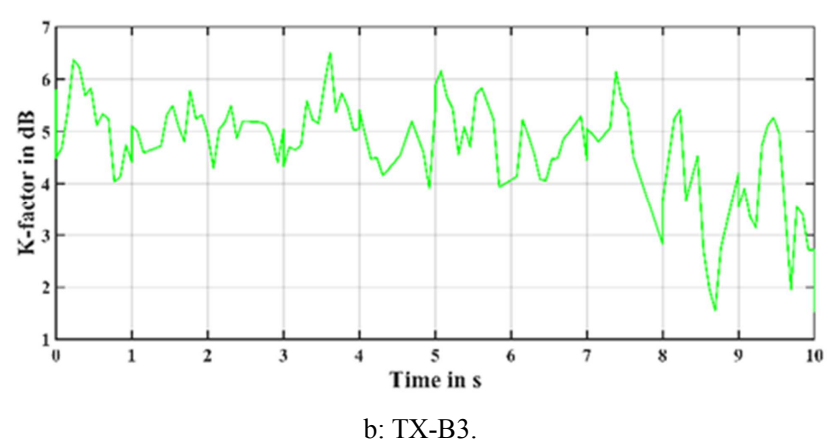

Figure 8. Test scenario Rician distribution K-factor.

\subsection{Power Delay Profile}

PDP is widely used to calculate the average dispersion of a transmitted signal in the time-delay domain. The term PDP refers to the power at the channel output as a function of the time delay. Previous research has established that the average and normalized PDP over a region can be regarded as stationary in the wide sense stationary (WSS). The wide sense stationary-uncorrelated scattering (WSSUS) model [23] has been applied to calculate the average delay and the root-mean-squared (RMS) delay spread extensively as a description of the frequency selection. To fully meet the requirements of the WSSUS, the time varying PDP [24] was calculated for the measurement data.

Using Equation (3), we obtained 1233 averaged PDPs for the 30 seconds measurement. These PDPs provided a measure of the spread of the received energy over time and were used to verify existence of multipath propagation. The different spread energies can also provide some information regarding the various motions in which the PDP was recorded.

Figure 9 presents an overview of the average PDPs from successive WSS regions for TX-B1. No mathematical expression could describe the shapes of the PDPs obtained from the measurements, and LOS propagation was evident. The LOS path was always dominant and strong. Meanwhile, a relatively weak path, labeled Path 1 , was parallel to the LOS component, which exhibited a long lifespan.

Using Equation (2) we obtained the entire PDP for the TX-B1 trajectory, as shown in Figure 9. The path power of the main strong path increased from -69.11 to $-53.05 \mathrm{~dB}$. The excess delays between the LOS and reflection (Path 1) paths in Figure 9 decrease by $0.42 \mu$ s from the beginning (excess delay: $2.34 \mu \mathrm{s}$ ) to the end (excess delay: $1.92 \mu \mathrm{s}$ ). The strong LOS path with a decreased delay indicated that the TX ship was moving near the RX station. The reflecting objects included specular reflections off the waterway wave surfaces, riverside buildings, and diffuse components from reflections off other objects surrounding the riverside, all of which could result in signal delays. Thus, LOS propagation dominated the delay domain this waterway, and the problems associated with weak reflections are present.

In the TX-B2 case, the path power of the main strong path was stable in the -72.95 to $-65.73 \mathrm{~dB}$ range. In this period, the excess delays between the LOS and reflection (Path 2) paths in Figure 10 increased by $0.05 \mu$ s from the beginning (excess delay: $0.54 \mu \mathrm{s}$ ) to the end (excess delay: $0.59 \mu \mathrm{s}$ ). Measurements were obtained when the ship was moving under the bridge. The distance between the RX and the bridge was 790 meters, based on GPS measurements, Google Maps, and the test videos. After the TX ship moved far from the RX and traveled under the bridge, a reflection path (Path 3 ) was evident in the $8-10 \mathrm{~s}$ range. In this period, the excess delays decreased by $0.07 \mu \mathrm{s}$ from the delay at $8 \mathrm{~s}$ (excess delay: $2.75 \mu \mathrm{s}$ ) to the delay at $10 \mathrm{~s}$ (excess delay: $2.68 \mu \mathrm{s}$ ). According to Google Maps and the test videos, other factors affected the communication between the TX and RX. Another important finding was that the excess delay difference was only 9 ns between the moment the ship began to move under the bridge (excess delay: $553 \mathrm{~ns}$ ) and the moment it finished (excess delay: $562 \mathrm{~ns}$ ). This excess delay difference $(9 \mathrm{~ns})$ was only $18 \%$ of the entire period $(0-10 \mathrm{~s}$, with an excess delay difference of $50 \mathrm{~ns}$ ), which indicated that the obstruction of the bridge affected the excess delays only slightly.

Compared with TX-B2, the main path in TX-B3 was in the -57.26 to $-65.54 \mathrm{~dB}$ range. During this period, the excess delays between the LOS and the reflection (Path 4) paths in Figure 10 increased by $0.3 \mu \mathrm{s}$ from the beginning (excess delay: $4.69 \mu \mathrm{s}$ ) to the end (excess delay: $4.99 \mu \mathrm{s}$ ). The excess delay of the ship under the bridge was similar to that of TX-B2. The excess delay difference was only $47 \mathrm{~ns}$ between the moment when the ship began to move under the bridge (excess delay: $4830 \mathrm{~ns}$ ) and the moment it finished (excess delay: 4887 ns). Conclusions from the excess delay difference analysis were similar to that from TX-B2. The excess delay difference $(47 \mathrm{~ns})$ was less than $16 \%$ of the entire period $(0-10 \mathrm{~s}$, with an excess delay difference of 300 ns). This indicated that the variation of the excess delays when the ship was under the bridge was small.

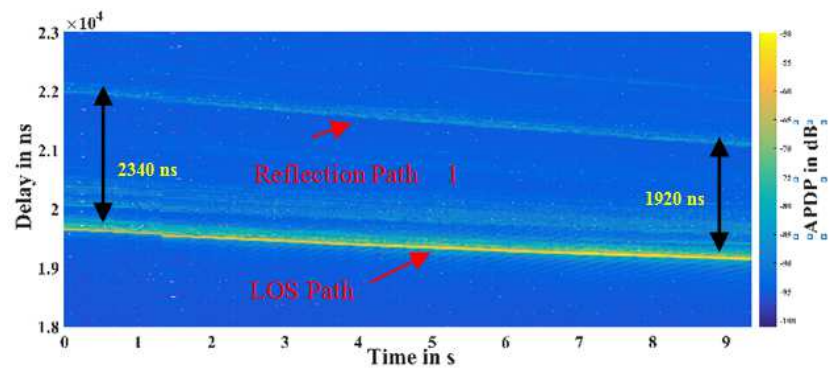

Figure 9. $P D P$ in $T X-B 1$ state.

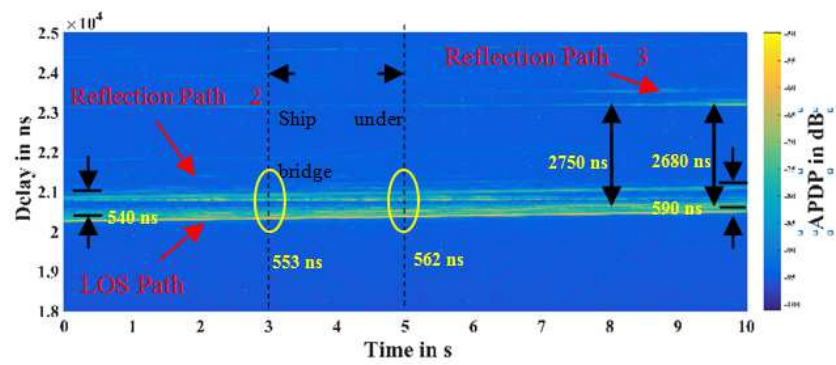

Figure 10. $P D P$ in $T X-B 2$ state. 


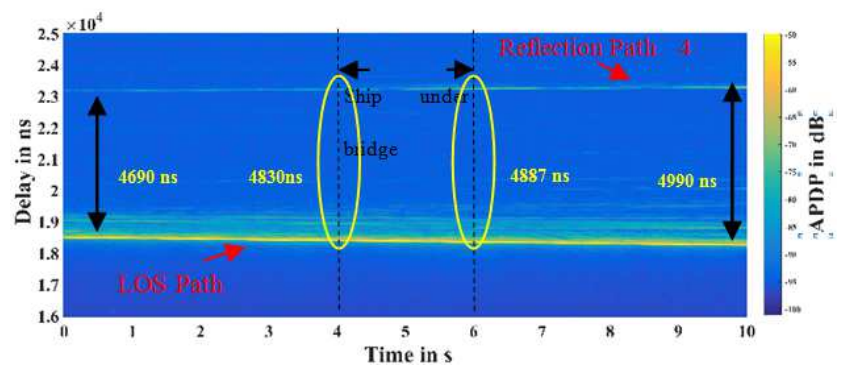

Figure 11. $P D P$ in $T X-B 3$ state.

The power of the signal was determined through the relationships between TX-B1, TX-B2, and TX-B3. In all cases, the direct signal power was the strongest when TX was closest to $\mathrm{RX}$. Furthermore, the signal was strong when there was a direct path between the RX and TX. This result contradicts previous studies that suggested that the LOS path loss over larger distances weakly affected the reception quality when a ship is approaching a bridge or moving on a river [14]. Obstructions and the surface of the water influence the received signal not only through propagation loss but also by acting as reflection points, which contributes extra power in the absence of LOS.

\subsection{Delay Spread}

The delay spread is the difference between the last resolvable delay signal and the arrival time of the earliest delay signal [15]. The signal arriving at the receiver is a composite of each signal that has a different path and a time difference. Thus, the composite signal will exhibit a delay spread relative to the original signal in the time domain. The delay spread generally describes the temporal dispersion characteristics of a multipath channel. The root mean square delay spread is the square root of the second order matrix of the power delay distribution of the multipath channel.

Due to the delay spread, the waveform of one symbol in the received signal is spread to other symbol periods, causing crosstalk between signals. To avoid such inter-symbol interference, the symbol period should be greater than the delay spread caused by the multipath effect. The mean delay and RMS delay spread are important parameters of the channel characteristics and often use the statistical coherence bandwidth.

The RMS delay spread describes the statistical characteristics of the multipath channel delay (11):

$$
\Delta^{2}=\int_{0}^{\infty}\left(\tau-\tau_{a}\right)^{2} P(\tau) d \tau
$$

where $\tau_{\alpha}$ is the average delay, and $\Delta$ is the mean squared delay spread, which characterizes the extent of the delay spread.

Figure 12 shows the probability density function (PDF) and cumulative density function (CDF) of the RMS delay spread for each case. The results were somewhat expected. The lowest delay spreads were found in the TX-B1 group, mostly because of its low density of scatterers around the route of moving ship. There were similarities between TX-B2 and TX-B3. TX-B2 exhibited the largest delay spreads of the three groups, possibly due to the ship motion direction and other far scatterers. The mean values of the delay spread with the typical values for each case are shown in Table 7.

Previous studies of the RMS delay spread values have not dealt with the UBE. The generalizability of much of the previously published research on this issue is unknown. The RMS delay spread results presented herein for low and high densities of scatterers will serve as a reference for inland waterway bridge environments. The probability of a 0 ns RMS delay spread for the TX-B1 case exceeded $70 \%$ because the dominant component was LOS propagation, whereas the delay spread was approximately 8 ns. Moreover, the RMS delay spread values for TX-B2 and TX-B3 were similar. The RMS delay in TX-B2 experienced the most severe multipath effects. The probability of a 0 ns RMS delay spread of the TX-B2 case was $90 \%$ because the dominant component was LOS propagation, as shown in Figure 12 (b). Similarly, the probability of a 0 ns RMS delay spread was $90 \%$ in the TX-B3 case, as shown in Figure 12c. Thus, the LOS component played a leading role in the propagation processes for TX-B2 and TX-B3. Although the time-dispersive features changed dramatically in the UBE, TX-B3 exhibited a higher RMS delay than TX-B2.
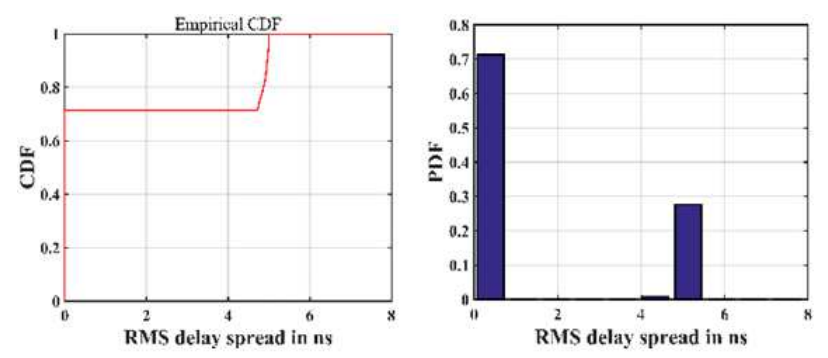

a
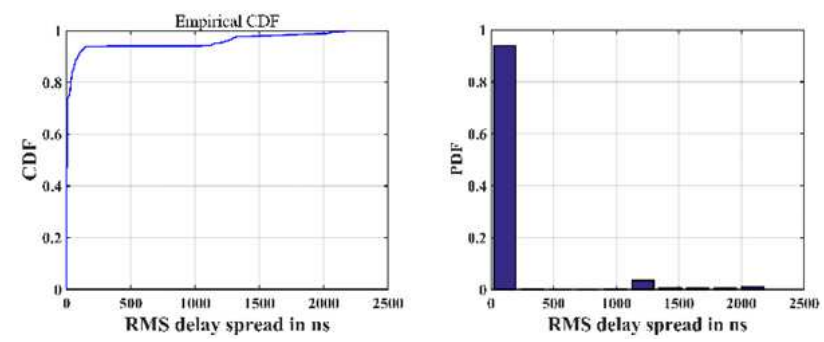

b
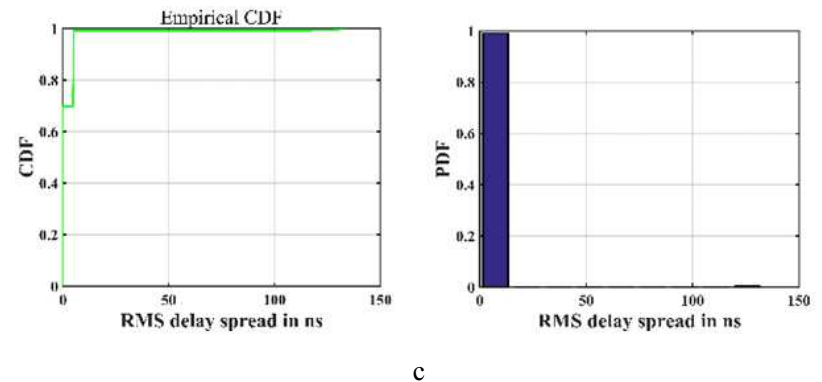

Figure 12. Comparison of the CDF and PDF of the RMS delay spread and mean delay spread in two different motion states.

CDF: cumulative density function.

PDF: probability density function.

(a) depict the CDF and PDF of TX-B1 state.

(b) depict the CDF and PDF of TX-B2 state.

(c) depict the CDF and PDF of TX-B3 state. 
Table 7. RMS Delay spread parameters for each investigated states.

\begin{tabular}{llll}
\hline & Min [ns] & Max [ns] & Mean [ns] \\
\hline TX-B1 & 0.37 & 7.89 & 1.408 \\
TX-B2 & 0.75 & 2202 & 102.177 \\
TX-B3 & 0.56 & 133.18 & 62.10 \\
\hline
\end{tabular}

Contrary to expectations, a significant difference between the UBS and LOS was not found in this study unless the ships were travelling in different directions. This indicates that when the ship moved under the bridge, reflection and blocking have a weak influence on signal propagation, and this moving state situation is different from other measurements in typical LOS scenarios. By comparison, the RMS delay spread value of the UBE was greater than the vehicle-to-vehicle delay spread in an urban environment [25].

\section{Conclusions}

In this investigation, the first aim was to assess the channel characteristics of an UBE for ship different motion states. UBE channel fading analysis and channel characteristics at $5.9 \mathrm{GHz}$ were comprehensively evaluated. Based on the distance change between the TX and RX, fundamental propagation mechanisms were investigated. The second aim of this study was to investigate the effects of the UBE for channel fading. The most significant finding was that the effects of the channel fading characteristics were not significantly different in UBE. The characteristics of channel fading for a moving ship in UBE were analyzed, as were the path loss and small-scale fading characteristics based on the ship moving. Before these measurements, the influence of urban bridges was purely anecdotal. This study is the first comprehensive investigation of ships in UBE. The bridge obstruction only slightly affected the excess delays of the wireless propagation. The values of the excess delay difference under the bridge were less than $20 \%$ of the entire measurement period. The standard deviation of the path loss differed greatly depending on when the test ship reached the minimum value or arrived at the bridge. The estimated RMS delay spread varied from 60 to $100 \mathrm{~ns}$ on average when the ships moved under the bridge compared to a ship in LOS.

\section{Acknowledgements}

The authors would like to express their sincere thanks to the Super Radio AS Oslo Norway for providing their $5.9 \mathrm{GHz}$ measurement equipment. The authors also appreciate for the supports of the Wuhan University of Technology. This work is supported by Young Scientists Fund of National Natural Science Foundation of China (no. 61701356).

\section{References}

[1] Tao Guo., "Key technology of Changjiang intelligent waterway," Port \& Waterway Engineering, 2016, (01): 99-105.

[2] QIU Wenqin; TANG Cunbao; TANG Qiangrong., "Navigation Environment Risk Assessment of Uncertain Inland Waterway,"
Navigation of China, 2019, (01): P52-55.

[3] Yu Song MENG, Yee Hui LEE, "Empirical modeling of ducting effects on a mobile microwave link over a sea surface," Radioengineering, 2012.

[4] Sumayya Balkees P A, Kalyan Sasidhar, "A Survey based Analysis of Propagation Models Over the Sea, International Conference on Advances in Computing, Communications and Informatics (ICACCI), 2015.

[5] QIU Wenqin; TANG Cunbao; TANG Qiangrong., "Status and prospect of technical development for bridges in China," Chinese Science Bulletin, 2016, V61: P 415-425.

[6] A. F. Molisch, F. Tufvesson, J. Karedal, and C. Mecklenbrauker, "A survey on vehicle-to-vehicle propagation channels," IEEE Commun. Lett., vol. 16, no. 6, pp. 12-22, Dec. 2009.

[7] A. F. Molisch, Wireless Communications, IEEE Press-Wiley, 2005.

[8] JQ Chen. A non-stationary channel model for 5G massive MIMO systems [J]. Frontiers of Information Technology \& Electronic Engineering, 2017. (12): 2101-2111.

[9] Junyi Yu.. Measurement-Based Characteristics Estimation of Radio Channel for Urban Campus Environment at 5.9 GHz. [J]. The Institution of Engineering and Technology, 2015. 1-9.

[10] JY. Chen. Method of the Recommendation ITU-R P. 1546-3 for VHF Field-Strength Prediction over sea propagation. Marine Technology, Vol, 3, April 2009, pp. 39-42.

[11] Reyes-Guerrero, J. C., \& Mariscal, L. A. (2012). Experimental time dispersion parameters of wireless channels over sea at 5.8 GHz. In Proceedings of IEEE international symposium on ELMAR, (pp. 89-92).

[12] Ruisi He, Andreas F. Molisch, Fredrik Tufvesson. Vehicle-to-Vehicle Propagation Models With Large Vehicle Obstructions [J]. IEEE Transactions on Intelligent Transportation Systems, 2014. 15 (5): 2237-2248.

[13] Song Xin, CQ Yuan. Inluence Analysis of Water Flow Rate on Operating Efficiency of Inland ships. Ship Engineering, Vol. 38 No. 72016 P: 54-57.

[14] P. Bello, "Characterization of randomly time-variant linear channels,"IEEE Trans. Commun. Syst., vol. CS-11, no. 4, pp. 360-393, Dec. 1963.

[15] Vitaly A. Karbolin, Vladimir I. Nosov. Performance Analysis of Ultra Wide Band Communication System in Terms of Data Rate Dependency and Sampling Rate Dependency of an Indoor Wireless Channel Impulse Response [J]. 14th International Scientific-Technical Conference APEIE, 2018. (): 184-187.

[16] Taimoor Abbas, Katrin Sjöberg, Johan Karedal, and Fredrik Tufvesson. A Measurement Based Shadow Fading Model for Vehicle-to-Vehicle Network Simulations [J]. International Journal of Antennas and Propagation, 2015. (): 1-12.

[17] Vlastaras D, Abbas T, Nilsson M, et al. Impact of a truck as an obstacle on vehicle-to-vehicle communications in rural and highway scenarios [C]//IEEE, International Symposium on Wireless Vehicular Communications. IEEE, 2014: 1-6,

[18] J.-M. Molina-Garcia-Pardo; M. Lienard. Wideband analysis of large scale and small scale fading in tunnels [J]. International Conference on ITS Telecommunications, Thailand, 2009. (5): 1-4. 
[19] H. Akaike, "Information theory and an extension of the maximum likelihood principle," in Selected Papers of Hirotugu Akaike. Springer, 1998, pp. 199-213.

[20] Haikuan Feng; Haojie Pei; Fuqin Yang. Estimation of leaf nitrogen content of maize based on Akaike's information criterion in Beijing [J]. IEEE International Geoscience and Remote Sensing Symposium (IGARSS), 2017. (7): 5057-5060.

[21] Jie Ding; Vahid Tarokh; Yuhong Yang. Bridging AIC and BIC: A New Criterion for Autoregression [J]. IEEE Transactions on Information Theory, 2018. 64 (6): 4024-4043.

[22] Y. Zhang and Y. Yang, "Cross-validation for selecting a model selection procedure," J. Econometrics, vol. 187, no. 1, pp. 95$112,2015$.
[23] C. A. Guti'errez, M. P atzold, W. Dahech, and N. Youssef, "A non-WSSUS mobile-to-mobile channel model assuming velocity variations of the mobile stations," in Proc. IEEE Wireless Commun. Netw. Conf., San Francisco, CA, USA, 2017, pp. 1-6.

[24] W. Dahech, M. P"atzold, C. A. Guti'errez, and N. Youssef, "A non-stationary mobile-to-mobile channel model allowing for velocity and trajectory variations of the mobile stations," IEEE Trans. Wireless Commun., vol. 16, no. 3, pp. 1987-2000, Mar. 2017

[25] L. Bernado, T. Zemen, F. Tufvesson, A. F. Molisch, and C. F. Mecklenbr "auker, "Delay and Doppler spreads of nonstationary vehicular channels for safety-relevant scenarios," IEEE Trans. Veh. Technol., vol. 63, no. 1, pp. 82-93, Jan. 2014. 